\title{
NEVANLINNA'S FIVE-VALUE THEOREM FOR DERIVATIVES OF ALGEBROID FUNCTIONS ON ANNULI
}

\author{
ASHOK RATHOD
}

\begin{abstract}
In this paper, we first obtain the famous Xiong Inequality for algebroid functions on annuli and also generalise Nevanlinna's five-value theorem for derivatives of algebroid functions by considering weaker assumptions of sharing five values and small functions to partially sharing $k(\geq 5)$ values and small functions on annuli. As a particular cases of our results, we deduce several results.
\end{abstract}

\section{Introduction}

The uniqueness theory of algebroid functions is an interesting problem in the value distribution theory. The uniqueness problem of algebroid functions was firstly considered by Valiron, afterwards some scholars have got several uniqueness theorems of algebroid functions in the complex plane $\mathbb{C}$ (see $[2,3,5,9,10,11]$ ). In 2005, A. Ya. Khrystiyanyn and A. A. Kondratyuk have proposed on the Nevanlinna Theory for meromorphic functions on annuli (see [7, 8]). In 2009, Cao and Yi [1] investigated the uniqueness of meromorphic functions sharing some values on annuli. In 2015,Yang Tan [12], Yang Tan and Yue Wang [13] proved some interesting results on the multiple values and uniqueness of algebroid functions on annuli. Thus it is interesting to consider the uniqueness problem of algebroid functions in multiply connected domains. By Doubly connected mapping theorem [17] each doubly connected domain is conformally equivalent to the annulus $\{z: r<|z|<R\}, 0 \leq r<R \leq+\infty$. We consider only two cases : $r=0, R=+\infty$ simultaneously and $0 \leq r<R \leq+\infty$. In the latter case the homothety $z \mapsto \frac{z}{r R}$ reduces the given domain to the annulus $\mathbb{A}\left(\frac{1}{R_{0}}, R_{0}\right)=\left\{z: \frac{1}{R_{0}}<|z|<R_{0}\right\}$, where $R_{0}=\sqrt{\frac{R}{r}}$. Thus, in two cases every annulus is invariant with respect to the inversion $z \mapsto \frac{1}{z}$

Received April 6, 2017, accepted November 30 , 2017.

2010 Mathematics Subject Classification. 30D35.

Key words and phrases. Value distribution theory, Nevanlinna theory, algebroid functions, sharing values and uniqueness, annuli. 


\section{Basic notations and definitions}

We assume that the reader is familiar with the Nevanlinna theory of meromorphic functions and algebroid functions (see [4] and [15]).

Let $A_{v}(z), A_{v-1}(z), \ldots, A_{0}(z)$ be a group of analytic functions which have no common zeros and define on the annulus $\mathbb{A}\left(\frac{1}{R_{0}}, R_{0}\right)\left(1<R_{0} \leq+\infty\right)$,

$$
\psi(z, W)=A_{v}(z) W^{\nu}+A_{\nu-1}(z) W^{\nu-1}+\cdots+A_{1}(z) W+A_{0}(z)=0 .
$$

Then irreducible equation (2.1) defines a $v$-valued algebroid function on the annulus $\mathbb{A}\left(\frac{1}{R_{0}}, R_{0}\right)$ $\left(1<R_{0} \leq+\infty\right)$.

Let $W(z)$ be a $v$-valued algebroid function on the annulus $\mathbb{A}\left(\frac{1}{R_{0}}, R_{0}\right)\left(1<R_{0} \leq+\infty\right)$, we use the following notations

$$
\begin{aligned}
& m(r, W)=\frac{1}{v} \sum_{j=1}^{v} m\left(r, w_{j}\right)=\frac{1}{v} \sum_{j=1}^{v} \frac{1}{2 \pi} \int_{0}^{2 \pi} \log ^{+}\left|w_{j}\left(r e^{i \theta}\right)\right| d \theta, \\
& N_{1}(r, W)=\frac{1}{v} \int_{\frac{1}{r}}^{1} \frac{n_{1}(t, W)}{t} d t, \quad N_{2}(r, W)=\frac{1}{v} \int_{1}^{r} \frac{n_{2}(t, W)}{t} d t, \\
& \bar{N}_{1}\left(r, \frac{1}{W-a}\right)=\frac{1}{v} \int_{\frac{1}{r}}^{1} \frac{\bar{n}_{1}\left(t, \frac{1}{W-a}\right)}{t} d t, \quad \bar{N}_{2}\left(r, \frac{1}{W-a}\right)=\frac{1}{v} \int_{1}^{r} \frac{\bar{n}_{2}\left(t, \frac{1}{W-a}\right)}{t} d t, \\
& m_{0}(r, W)=m(r, W)+m\left(\frac{1}{r}, W\right)-2 m(1, W), N_{0}(r, W)=N_{1}(r, W)+N_{2}(r, W), \\
& \bar{N}_{0}\left(r, \frac{1}{W-a}\right)=\bar{N}_{1}\left(r, \frac{1}{W-a}\right)+\bar{N}_{2}\left(r, \frac{1}{W-a}\right),
\end{aligned}
$$

where $w_{j}(z)(j=1,2, \ldots, v)$ is one valued branch of $W(z), n_{1}(t, W)$ is the counting function of poles of the function $W(z)$ in $\{z: t<|z| \leq 1\}$ and $n_{2}(t, W)$ is the counting function of poles of the function $W(z)$ in $\{z: 1<|z| \leq t\}$ (both counting multiplicity). $\bar{n}_{1}\left(t, \frac{1}{W-a}\right)$ is the counting function of poles of the function $\frac{1}{W-a}$ in $\{z: t<|z| \leq 1\}$ and $\bar{n}_{2}\left(t, \frac{1}{W-a}\right)$ is the counting function of poles of the function $\frac{1}{W-a}$ in $\{z: 1<|z| \leq t\}$ (both ignoring multiplicity). $\bar{n}_{1}^{k)}\left(t, \frac{1}{W-a}\right)\left(\bar{n}_{1}^{(k}\left(t, \frac{1}{W-a}\right)\right)$ is the counting function of poles of the function $\frac{1}{W-a}$ with multiplicity $\leq k$ (or $>k$ ) in $\{z: t<|z| \leq 1\}$, each point count only once; $\bar{n}_{2}^{k)}\left(t, \frac{1}{W-a}\right)\left(\bar{n}_{2}^{(k}\left(t, \frac{1}{W-a}\right)\right)$ is the counting function of poles of the function $\frac{1}{W-a}$ with multiplicity $\leq k$ (or $>k$ ) in $\{z: 1<$ $|z| \leq t\}$, each point count only once, respectively.

Let $W(z)$ be a $v$-valued algebroid function which determined by (2.1) on the annulus $\mathbb{A}\left(\frac{1}{R_{0}}, R_{0}\right)\left(1<R_{0} \leq+\infty\right)$, when $a \in \mathbb{C}, n_{0}\left(r, \frac{1}{W-a}\right)=n_{0}\left(r, \frac{1}{\psi(z, a)}\right), N_{0}\left(r, \frac{1}{W-a}\right)=\frac{1}{v} N_{0}\left(r, \frac{1}{\psi(z, a)}\right)$. In particular, when $a=0, N_{0}\left(r, \frac{1}{W}\right)=\frac{1}{v} N_{0}\left(r, \frac{1}{A_{0}}\right)$. When $a=\infty, N_{0}(r, W)=\frac{1}{v} N_{0}\left(r, \frac{1}{A_{v}}\right)$; where $n_{0}\left(r, \frac{1}{W-a}\right)$ and $n_{0}\left(r, \frac{1}{\psi(z, a)}\right)$ are the counting function of zeros of $W(z)-a$ and $\psi(z, a)$ on the annulus $\mathbb{A}\left(\frac{1}{R_{0}}, R_{0}\right)\left(1<R_{0} \leq+\infty\right)$, respectively. 
Definition 2.1 ([12]). Let $W(z)$ be an algebroid function on the annulus $\mathbb{A}\left(\frac{1}{R_{0}}, R_{0}\right)\left(1<R_{0} \leq\right.$ $+\infty)$, the function

$$
T_{0}(r, W)=m_{0}(r, W)+N_{0}(r, W), \quad 1 \leq r<R_{0}
$$

is called Nevanlinna characteristic of $W(z)$.

\section{Some lemmas}

Lemma 3.1 ([7] (Jensen theorem for meromorphic function on annuli)). Let $f$ be a meromorphic function on the annulus $\mathbb{A}\left(\frac{1}{R_{0}}, R_{0}\right)\left(1<R_{0} \leq+\infty\right)$, then

$$
\begin{aligned}
N_{0}\left(r, \frac{1}{f}\right)-N_{0}(r, f)= & \frac{1}{2 \pi} \int_{0}^{2 \pi} \log \left|f\left(r e^{i \theta}\right)\right| d \theta+\frac{1}{2 \pi} \int_{0}^{2 \pi} \log \left|f\left(\frac{1}{r} e^{i \theta}\right)\right| d \theta \\
& -\frac{1}{2 \pi} \int_{0}^{2 \pi} \log \left|f\left(e^{i \theta}\right)\right| d \theta,
\end{aligned}
$$

where $1 \leq r<R_{0}$.

Lemma 3.2 ([13] (The first fundamental theorem on annuli)). Let $W(z)$ be $v$-valued algebroid function which is determined by (2.1) on the annulus $\mathbb{A}\left(\frac{1}{R_{0}}, R_{0}\right)\left(1<R_{0} \leq+\infty\right), a \in \mathbb{C}$

$$
m_{0}(r, a)+N_{0}(r, a)=T_{0}(r, W)+O(1)
$$

Lemma 3.3 ([13] (The second fundamental theorem on annuli)). Let $W(z)$ be v-valued algebroid function which is determined by (2.1) on the annulus $\mathbb{A}\left(\frac{1}{R_{0}}, R_{0}\right)\left(1<R_{0} \leq+\infty\right), a_{k}(k=$ $1,2, \ldots, p)$ are $p$ distinct complex numbers (finite or infinite), then we have

$$
(p-2 v) T_{0}(r, W) \leq \sum_{k=1}^{p} N_{0}\left(r, \frac{1}{W-a_{k}}\right)-N_{1}(r, W)+S_{0}(r, W)
$$

$N_{1}(r, W)$ is the density index of all multiple values including finite or infinite, every $\tau$ multiple value counts $\tau-1$, and

$$
S_{0}(r, W)=m_{0}\left(r, \frac{W^{\prime}}{W}\right)+\sum_{j=1}^{p} m_{0}\left(r, \frac{W^{\prime}}{W-a_{k}}\right)+O(1) .
$$

The remainder of the second fundamental theorem is the following formula

$$
S_{0}(r, W)=O\left(\log T_{0}(r, W)\right)+O(\log r)
$$

outside a set of finite linear measure, if $r \rightarrow R_{0}=+\infty$, while

$$
S_{0}(r, W)=O\left(\log T_{0}(r, W)\right)+O\left(\log \frac{1}{R_{0}-r}\right),
$$

outside a set $E$ of $r$ such that $\int_{E} \frac{d r}{R_{0}-r}<+\infty$, when $r \rightarrow R_{0}<+\infty$. 
Remark 3.1 ([13]). The second fundamental theorem on annuli has other forms, as the following:

$$
\begin{gathered}
(p-1) T_{0}(r, W) \leq N_{0}(r, W)+\sum_{k=1}^{p} N_{0}\left(r, \frac{1}{W-a_{k}}\right)-N_{1}(r)+Q_{1}(r, W), \\
N_{1}(r, W)=2 N_{0}(r, W)-N_{0}\left(r, W^{\prime}\right)+N_{0}\left(r, \frac{1}{W^{\prime}}\right), \\
Q_{1}(r, W)=\sum_{k=0}^{p} m_{0}\left(r, \frac{W^{\prime}}{W-a_{k}}\right)+O(1), a_{0}=0 .
\end{gathered}
$$

We notice that the following formula is true,

$$
\sum_{k=1}^{p} N_{0}\left(r, \frac{1}{W-a_{k}}\right)-N_{1}(r, W) \leq \sum_{k=1}^{p} \bar{N}_{0}\left(r, \frac{1}{W-a_{k}}\right) .
$$

$\bar{N}_{0}\left(r, \frac{1}{W-a_{k}}\right)$ is the reduced counting function of zeros(ignoring multiplicity). Then the second fundamental theorem can be rewritten as the following

$$
(p-2 v) T_{0}(r, W) \leq \sum_{k=1}^{p} \bar{N}_{0}\left(r, \frac{1}{W-a_{k}}\right)+S_{0}(r, W) .
$$

Lemma 3.4 ([13]). Let $W(z)$ be v-valued algebroid function which is determined by (2.1) on the annulus $\mathbb{A}\left(\frac{1}{R_{0}}, R_{0}\right)\left(1<R_{0} \leq+\infty\right)$, if the following conditions are satisfied

$$
\begin{aligned}
& \underset{r \rightarrow \infty}{\lim } \frac{T_{0}(r, W)}{\log r}<\infty, \quad R_{0}=+\infty, \\
& \underset{r \rightarrow R_{0}^{-}}{\log \frac{T_{0}(r, W)}{\left(R_{0}-r\right)}<\infty,} \quad R_{0}<+\infty,
\end{aligned}
$$

then $W(z)$ is an algebraic function.

Remark 3.2 ([13]). Let $W(z)$ be a $v$-valued algebroid function which is determined by (2.1) on the annulus $\mathbb{A}\left(\frac{1}{R_{0}}, R_{0}\right)$, where $1<R_{0} \leq+\infty$ and $\widehat{W}(z)$ be a $\mu$-valued algebroid functions which is determined by the following equation on the annulus $\mathbb{A}\left(\frac{1}{R_{0}}, R_{0}\right)$, where $1<R_{0} \leq+\infty$,

$$
\varphi(z, \widehat{W})=B_{\mu}(z) \widehat{W}^{\mu}+B_{\mu-1}(z) \widehat{W}^{\mu-1}+\cdots+B_{1}(z) \widehat{W}+B_{0}(z)=0 .
$$

Without loss of generality, let $\mu \leq v, \bar{n}_{\Delta}(r, a)$ denotes the counting function of the common values of $W(z)=a$ and $\widehat{W}(z)=a$ on the annulus $\mathbb{A}\left(\frac{1}{R_{0}}, R_{0}\right)\left(1<R_{0} \leq+\infty\right)$, ignoring multiplicity. And let

$$
\begin{aligned}
& \bar{N}_{\Delta}(r, a)=\frac{\mu+v}{2 \mu \nu} \int_{\frac{1}{r}}^{1} \frac{\bar{n}_{\Delta_{1}}(t, a)}{t} d t+\frac{\mu+v}{2 \mu \nu} \int_{1}^{r} \frac{\bar{n}_{\Delta_{2}}(t, a)}{t} d t \\
& \bar{N}_{12}(r, a)=\bar{N}_{0}\left(r, \frac{1}{W-a}\right)+\bar{N}_{0}\left(r, \frac{1}{\widehat{W}-a}\right)-2 \bar{N}_{\Delta}(r, a) .
\end{aligned}
$$




\section{Main results}

Let $W(z)$ be an algebroid function on the annulus $\mathbb{A}\left(\frac{1}{R_{0}}, R_{0}\right)$, where $1<R_{0} \leq+\infty$, and $a$ be a complex number in the extended complex plane. Write $E(a, W)=\{z \in \mathbb{A}: W(z)-a=$ $0\}$, where each zero with multiplicity $m$ is counted $m$ times. If we ignore the multiplicity, then the set is denoted by $\bar{E}(a, W)$. We use $\bar{E}_{k)}(a, W)$ to denote the set of zeros of $W-a$ with multiplicities not greater than $k$, in which each zero is counted only once.

In this paper, we say that two algebroid functions on the annulus $\mathbb{A}\left(\frac{1}{R_{0}}, R_{0}\right)\left(1<R_{0} \leq\right.$ $+\infty)$, share a function $a(z)$ if we have $W(z)-a(z)=0$ if and only if $\widehat{W}-a(z)=0$. Now we consider the case that two algebroid function partially share small functions.

Definition 4.1. Let $W(z)$ be an algebroid function on the annulus $\mathbb{A}\left(\frac{1}{R_{0}}, R_{0}\right)\left(1<R_{0} \leq+\infty\right)$ and $a(z)$ be a small function of $W(z)$. We define

$$
\bar{W}(a, W)=\{z \mid W(z)-a(z)=0\}
$$

in which each zero is counted only once.

We say that an algebroid function $W(z)$ partially shares a value $a$ with an algebroid function $\widehat{W}(z)$ on the annulus $\mathbb{A}\left(\frac{1}{R_{0}}, R_{0}\right)\left(1<R_{0} \leq+\infty\right)$ if

$$
\bar{E}(a, W) \subseteq \bar{E}(a, \widehat{W})
$$

To prove our main theorem, we need to get the following Xiong inequality for algebroid functions on annuli.

Theorem 4.1. Let $W(z)$ be a v-valued algebroid function determined by (2.1) on the annulus $\mathbb{A}\left(\frac{1}{R_{0}}, R_{0}\right)\left(1<R_{0} \leq+\infty\right)$, respectively and $b_{j}(j=1,2, \ldots, q)$ be distinct finite non zero complex numbers. Then for any positive integer $n$, we have

$$
\begin{aligned}
q T_{0}(r, W)< & \bar{N}_{0}(r, W)+q N_{0}\left(r, \frac{1}{W}\right)+\sum_{j=1}^{q} N_{0}\left(r, \frac{1}{W^{(n)}-b_{j}}\right) \\
& -\left[(q-1) N_{0}\left(r, \frac{1}{W^{(n)}}\right)+N_{0}\left(r, \frac{1}{W^{(n+1)}}\right)\right]+S_{0}(r, W) .
\end{aligned}
$$

Proof. We have

$$
\begin{aligned}
T_{0}\left(r, W^{\prime}\right) & =T_{0}\left(r, W \frac{W^{\prime}}{W}\right) \leq T_{0}(r, W)+T_{0}\left(r, \frac{W^{\prime}}{W}\right)+O(1) \\
& \leq T_{0}(r, W)+m_{0}\left(r, \frac{W^{\prime}}{W}\right)+N_{0}\left(r, \frac{W^{\prime}}{W}\right)+O(1) \\
& =T_{0}(r, W)+\bar{N}_{0}(r, W)+S_{0}(r, W)
\end{aligned}
$$




$$
=2 v T_{0}(r, W)+S_{0}(r, W) .
$$

Hence, by Lemma 3.3 and (4.2), we have

$$
\begin{aligned}
S_{0}\left(r, W^{(k)}\right)=O\left(\log r T_{0}\left(r, W^{(k)}\right)\right) & =O\left(\log r T_{0}(r, W)\right)=S_{0}(r, W) . \\
m_{0}\left(r, \frac{W^{(k)}}{W-a_{i}}\right) & =S_{0}(r, W) .
\end{aligned}
$$

From Lemma 3.3, (4.3) and (4.4), we have

$$
m_{0}\left(r, \frac{W^{(k)}}{\prod_{i=1}^{p}\left(W-a_{i}\right)}\right)=S_{0}\left(r, W^{(k)}\right), \quad m_{0}\left(r, \frac{W^{(k+1)}}{W^{(k)} \prod_{j=1}^{q}\left(W^{(k)}-b_{j}\right)}\right)=S_{0}\left(r, W^{(k)}\right)
$$

and

$$
\frac{1}{\prod_{i=1}^{p}\left(W-a_{i}\right)^{n}}=\left\{\frac{W^{(k)}}{\prod_{i=1}^{p}\left(W-a_{i}\right)}\right\}^{n} \frac{W^{(k+1)}}{W^{(k)} \prod_{j=1}^{q}\left(W^{(k)}-b_{j}\right)} \frac{\prod_{j=1}^{q}\left(W^{(k)}-b_{j}\right)}{\left(W^{k}\right)^{n-1} W^{(k+1)}}
$$

Then

$$
n m_{0}\left(r, \frac{1}{\prod_{i=1}^{p}\left(W-a_{i}\right)}\right) \leq m_{0}\left(r, \frac{\prod_{j=1}^{q}\left(W^{(k)}-b_{j}\right)}{\left(W^{k}\right)^{n-1} W^{(k+1)}}\right)+S_{0}\left(r, W^{(k)}\right) .
$$

From (4.3), Lemma 3.1 and 3.3, we have

$$
\begin{aligned}
m_{0}\left(r, \frac{\prod_{j=1}^{q}\left(W^{(k)}-b_{j}\right)}{\left(W^{(k)}\right)^{n-1} W^{(k+1)}}\right)= & N_{0}\left(r, \frac{\left(W^{(k)}\right)^{n-1} W^{(k+1)}}{\prod_{j=1}^{q}\left(W^{(k)}-b_{j}\right)}\right)-N_{0}\left(r, \frac{\prod_{j=1}^{q}\left(W^{(k)}-b_{j}\right)}{\left(W^{(k)}\right)^{n-1} W^{(k+1)}}\right)+S_{0}\left(r, W^{(k)}\right) \\
= & \bar{N}_{0}(r, W)-(q-n) N_{0}\left(R, W^{(k)}\right)+\sum_{j=1}^{q} N_{0}\left(r, \frac{1}{W^{(k)}-b_{j}}\right) \\
& -(n-1) N_{0}\left(r, \frac{1}{W^{(k)}}\right)-N_{0}\left(r, \frac{1}{W^{(k+1)}}\right)+S_{0}\left(r, W^{(k)}\right) .
\end{aligned}
$$

From Lemma 3.1 and (4.2), (4.3), the left of (4.6) can be replaced by

$$
\begin{aligned}
n m_{0}\left(r, \frac{1}{\prod_{i=1}^{p}\left(W-a_{i}\right)}\right) & =n T_{0}\left(r, \prod_{i=1}^{p}\left(W-a_{i}\right)\right)-n N_{0}\left(r, \frac{1}{\prod_{i=1}^{p}\left(W-a_{i}\right)}\right)+O(1) \\
& =n p T_{0}(r, W)-n \sum_{i=1} p N_{0}\left(r, \frac{1}{\left(W-a_{i}\right)}\right)+S_{0}\left(r, W^{(k)}\right) .
\end{aligned}
$$


Put (4.6) and (4.7) into (4.5), then we have

$$
\begin{aligned}
n p T_{0}(r, W) \leq & \bar{N}_{0}(r, W)+n \sum_{i=1}^{p} N_{0}\left(r, \frac{1}{\left(W-a_{i}\right)}\right)+\sum_{j=1}^{q} N_{0}\left(r, \frac{1}{W^{(k)}-b_{j}}\right) \\
& -(q-n) N_{0}\left(r, W^{(k)}\right)-(n-1) N_{0}\left(r, \frac{1}{W^{(k)}}\right)-N_{0}\left(r, \frac{1}{W^{(k+1)}}\right)+S_{0}(r, W) .
\end{aligned}
$$

Let $n=q, p=1$, we can get the inequality (4.1). The proof of Theorem 4.1 is completed.

It is natural question to ask if $W^{(n)}(z)$ and $\widehat{W}^{(n)}(z)$ be two $v$-valued and $\mu$-valued algebroid functions on annulus $\mathbb{A}\left(\frac{1}{R_{0}}, R_{0}\right)\left(1<R_{0} \leq+\infty\right)$, partially share more than five values for a positive integer $n$, what the corresponding inequality becomes? Below we answer for this equations by proving the following theorem.

Theorem 4.2. Let $W(z)$ and $\widehat{W}(z)$ be two $v$-valued and $\mu$-valued algebroid functions determined by (2.1) on the annulus $\mathbb{A}\left(\frac{1}{R_{0}}, R_{0}\right)\left(1<R_{0} \leq+\infty\right)$, respectively and $\mu \leq v$, let $a_{j}(j=$ $1,2, \ldots, k)$ be $k$ distinct small functions, where $k \geq 4 v+1$ and for a non negative integers $n$, if

$$
\begin{gathered}
E\left(a_{j}, W^{(n)}\right) \subseteq E\left(a_{j}, \widehat{W}^{(n)}\right), \quad \text { forall } 1 \leq j \leq k, \\
E\left(0, W_{1}\right) \subseteq E\left(0, W^{(n)}\right) \text { and } E(0, \widehat{W}) \subseteq E\left(0, \widehat{W}^{(n)}\right),
\end{gathered}
$$

and

$$
\frac{\varliminf_{r \rightarrow \infty} \sum_{j=1}^{k} N_{0}\left(r, \frac{1}{W^{(n)}-a_{j}}\right)}{\underline{\lim _{r \rightarrow \infty}} \sum_{j=1}^{k} N_{0}\left(r, \frac{1}{\bar{W}^{(n)}-a_{j}}\right)}>\frac{n+1}{k-(n+2 v+1)},
$$

then $W^{(n)}(z) \equiv \widehat{W}^{(n)}(z)$.

Proof. Given $\in>0$ and from Theorem 4.1, we have

$$
\begin{aligned}
(k-2 v-\epsilon) T_{0}(r, W) \leq & \bar{N}_{0}(r, W)+(k-2 v) N_{0}\left(r, \frac{1}{W}\right)+\sum_{j=1}^{k-2 v} N_{0}\left(r, \frac{1}{W^{(n)}-a_{j}}\right) \\
& -(k-(2 v+1)) N_{0}\left(r, \frac{1}{W^{(n)}}\right)+S_{0}(r, W)
\end{aligned}
$$

and

$$
\begin{aligned}
(k-2 v-\epsilon) T_{0}(r, \widehat{W}) \leq & \bar{N}_{0}(r, \widehat{W})+(k-2 v) N_{0}\left(r, \frac{1}{\widehat{W}}\right)+\sum_{j=1}^{k-2 v} N_{0}\left(r, \frac{1}{\widehat{W}^{(n)}-a_{j}}\right) \\
& -(k-(2 v+1)) N_{0}\left(r, \frac{1}{\widehat{W}^{(n)}}\right)+S_{0}(r, \widehat{W}) .
\end{aligned}
$$


Using (4.9), (4.11) and (4.12) reduces to

$$
\begin{aligned}
(k-2 v-\epsilon) T_{0}(r, W) \leq & \bar{N}_{0}(r, W)+N_{0}\left(r, \frac{1}{W^{(n)}}\right) \\
& +\sum_{j=1}^{k-2 v} N_{0}\left(r, \frac{1}{W^{(n)}-a_{j}}\right)+S_{0}(r, W)
\end{aligned}
$$

and

$$
\begin{aligned}
(k-2 v-\epsilon) T_{0}(r, \widehat{W}) \leq & \bar{N}_{0}(r, \widehat{W})+N_{0}\left(r, \frac{1}{\widehat{W}^{(n)}}\right) \\
& +\sum_{j=1}^{k-2 v} N_{0}\left(r, \frac{1}{\widehat{W}^{(n)}-a_{j}}\right)+S_{0}(r, \widehat{W}) .
\end{aligned}
$$

Without loss of generality, we may assume $a_{k}=\infty$ and $a_{k-1}=0$.

First we may assume that all $a_{j}(1 \leq j \leq k)$ in (4.8) are finite. Then by (4.13) and (4.14), we have

$$
(k-(2 v+1)-\epsilon) T_{0}(r, W) \leq \sum_{j=1}^{k-1} N_{0}\left(r, \frac{1}{W^{(n)}-a_{j}}\right)+S_{0}(r, W)
$$

and

$$
(k-(2 v+1)-\epsilon) T_{0}(r, \widehat{W}) \leq \sum_{j=1}^{k-1} N_{0}\left(r, \frac{1}{\widehat{W}^{(n)}-a_{j}}\right)+S_{0}(r, \widehat{W}) .
$$

From (4.15), (4.16) and by Remark 3.2, we have

$$
\begin{aligned}
(q-(2 v+1)-\epsilon)\left[T_{0}(r, W)+T_{0}(r, \widehat{W})\right] \leq & \sum_{j=1}^{k-1} \bar{N}_{0}\left(r, \frac{1}{W^{(n)}-a_{j}}\right)+\sum_{j=1}^{q} \bar{N}_{0}\left(r, \frac{1}{\widehat{W}^{(n)}-a_{j}}\right) \\
& +S_{0}(r, W)+S_{0}(r, \widehat{W}), \\
\leq & \sum_{j=1}^{k-1} \bar{N}_{12}\left(r, a_{j}\right)+2 \sum_{j=1}^{k-1} \bar{N}_{\Delta}\left(r, a_{j}\right) \\
& +S_{0}(r, W)+S_{0}(r, \widehat{W}) .
\end{aligned}
$$

If $W^{(n)}(z) \not \equiv \widehat{W}^{(n)}(z)$, then we have

$$
\sum \bar{n}_{\Delta}(r, a) \leq n_{0}\left(r, \frac{1}{R(\varphi, \psi)}\right),
$$

$R(\varphi, \psi)$ denotes the resultant of $\varphi\left(z, W^{(n)}\right)$ and $\psi\left(z, W^{(n)}\right)$, it can be written as the following

$$
R(\varphi, \psi)=\left[A_{v}(z)\right]^{\mu}\left[B_{\mu}(z)\right]^{v} \prod_{\substack{1 \leq j \leq v \\ 1 \leq k \leq \mu}}\left[w_{j}^{(n)}(z)-\widehat{w}_{j}^{(n)}(z)\right] .
$$

It can be written in the another form

$$
R(\varphi, \psi)=\left|\begin{array}{cccccccc}
A_{v}(z) & A_{v-1}(z) & \ldots & \ldots & A_{0}(z) & 0 & \ldots & 0 \\
0 & A_{v}(z) & A_{v-1}(z) & \ldots & A_{1}(z) & A_{0}(z) & \ldots & 0 \\
\vdots & \vdots & & & \vdots & & & \\
0 & 0 & 0 & A_{v}(z) & A_{v-1}(z) & \ldots & \ldots & A_{0}(z) \\
B_{\mu}(z) & B_{\mu-1}(z) & \ldots & \ldots & B_{0}(z) & 0 & \ldots & 0 \\
0 & B_{\mu}(z) & B_{\mu-1}(z) & \ldots & B_{1}(z) & B_{0}(z) & \ldots & 0 \\
\vdots & \vdots & & & \vdots & & \\
0 & 0 & 0 & B_{\mu}(z) & B_{\mu-1}(z) & \ldots & \ldots & B_{0}(z)
\end{array}\right|
$$


So we know that $R(\varphi, \psi)$ is a holomorphic function and using Jensen Theorem for meromorphic function on annuli, we have

$$
\begin{aligned}
& N_{0}\left(r, \frac{1}{R(\varphi, \psi)}\right) \\
& =\frac{1}{2 \pi} \int_{0}^{2 \pi} \log \left|R\left[\psi\left(r e^{i \theta}, W^{(n)}\right), \varphi\left(r e^{i \theta}, \widehat{W}^{(n)}\right)\right]\right| d \theta \\
& +\frac{1}{2 \pi} \int_{0}^{2 \pi} \log \left|R\left[\psi\left(\frac{1}{r} e^{i \theta}, W^{(n)}\right), \varphi\left(\frac{1}{r} e^{i \theta}, \widehat{W}^{(n)}\right)\right]\right| d \theta \\
& +2 \frac{1}{2 \pi} \int_{0}^{2 \pi} \log \left|R\left[\psi\left(e^{i \theta}, W^{(n)}\right), \varphi\left(e^{i \theta}, \widehat{W}^{(n)}\right)\right]\right| d \theta \\
& =\frac{\mu}{2 \pi} \int_{0}^{2 \pi} \log \left|A_{v}\left(r e^{i \theta}\right)\right| d \theta+\frac{v}{2 \pi} \int_{0}^{2 \pi} \log \left|B_{\mu}\left(r e^{i \theta}\right)\right| d \theta \\
& +\frac{1}{2 \pi} \int_{0}^{2 \pi} \log \left|\prod_{\substack{1 \leq j \leq v \\
1 \leq k \leq \mu}}\left[w_{j}^{(n)}\left(r e^{i \theta}\right)-\widehat{w}_{j}^{(n)}\left(r e^{i \theta}\right)\right]\right| d \theta \\
& +\frac{\mu}{2 \pi} \int_{0}^{2 \pi} \log \left|A_{v}\left(\frac{1}{r} e^{i \theta}\right)\right| d \theta+\frac{v}{2 \pi} \int_{0}^{2 \pi} \log \left|B_{\mu}\left(\frac{1}{r} e^{i \theta}\right)\right| d \theta \\
& +\frac{1}{2 \pi} \int_{0}^{2 \pi} \log \left|\prod_{\substack{1 \leq j \leq v \\
1 \leq k \leq \mu}}\left[w_{j}^{(n)}\left(\frac{1}{r} e^{i \theta}\right)-\widehat{w}_{j}^{(n)}\left(\frac{1}{r} e^{i \theta}\right)\right]\right| d \theta-2 \cdot \frac{\mu}{2 \pi} \int_{0}^{2 \pi} \log \left|A_{v}\left(e^{i \theta}\right)\right| d \theta \\
& -2 \frac{v}{2 \pi} \int_{0}^{2 \pi} \log \left|B_{\mu}\left(e^{i \theta}\right)\right| d \theta-2 \frac{1}{2 \pi} \int_{0}^{2 \pi} \log \left|\prod_{\substack{1 \leq j \leq \nu \\
1 \leq k \leq \mu}}\left[w_{j}^{(n)}\left(e^{i \theta}\right)-\widehat{w}_{j}^{(n)}\left(e^{i \theta}\right)\right]\right| d \theta \\
& =\frac{\mu}{2 \pi} \int_{0}^{2 \pi} \log \left|A_{v}\left(r e^{i \theta}\right)\right| d \theta+\frac{\mu}{2 \pi} \int_{0}^{2 \pi} \log \left|A_{v}\left(\frac{1}{r} e^{i \theta}\right)\right| d \theta-2 \frac{\mu}{2 \pi} \int_{0}^{2 \pi} \log \left|A_{v}\left(e^{i \theta}\right)\right| d \theta \\
& +\frac{v}{2 \pi} \int_{0}^{2 \pi} \log \left|B_{\mu}\left(r e^{i \theta}\right)\right| d \theta+\frac{v}{2 \pi} \int_{0}^{2 \pi} \log \left|B_{\mu}\left(\frac{1}{r} e^{i \theta}\right)\right| d \theta-2 \frac{v}{2 \pi} \int_{0}^{2 \pi} \log \left|B_{\mu}\left(e^{i \theta}\right)\right| d \theta \\
& +\frac{1}{2 \pi} \int_{0}^{2 \pi} \log \left|\prod_{\substack{1 \leq j \leq v \\
1 \leq k \leq \mu}}\left[w_{j}^{(n)}\left(r e^{i \theta}\right)-\widehat{w}_{j}^{(n)}\left(r e^{i \theta}\right)\right]\right| d \theta \\
& +\frac{1}{2 \pi} \int_{0}^{2 \pi} \log \left|\prod_{\substack{1 \leq j \leq \nu \\
1 \leq k \leq \mu}}\left[w_{j}^{(n)}\left(\frac{1}{r} e^{i \theta}\right)-\widehat{w}_{j}^{(n)}\left(\frac{1}{r} e^{i \theta}\right)\right]\right| d \theta \\
& -2 \cdot \frac{1}{2 \pi} \int_{0}^{2 \pi} \log \left|\prod_{\substack{1 \leq j \leq \nu \\
1 \leq k \leq \mu}}\left[w_{j}^{(n)}\left(e^{i \theta}\right)-\widehat{w}_{j}^{(n)}\left(e^{i \theta}\right)\right]\right| d \theta
\end{aligned}
$$




$$
\begin{aligned}
\leq & \mu\left[m_{0}\left(r, A_{v}\right)-m_{0}\left(r, \frac{1}{A_{v}}\right)\right]+v\left[m_{0}\left(r, B_{\mu}\right)-m_{0}\left(r, \frac{1}{B_{\mu}}\right)\right] \\
& +\mu v\left[m_{0}\left(r, W^{(n)}\right)+m_{0}\left(r, \widehat{W}^{(n)}\right)\right]+O(1) \\
= & \mu v\left[T_{0}\left(r, W^{(n)}\right)+T_{0}\left(r, \widehat{W}^{(n)}\right)\right]+O(1) .
\end{aligned}
$$

Then we get

$$
\begin{aligned}
\sum \bar{N}_{\Delta}\left(r, a_{j}\right) & \leq \frac{2 \mu v}{\mu+v}\left[T_{0}\left(r, W^{(n)}\right)+T_{0}\left(r, \widehat{W}^{(n)}\right)\right]+O(1) \\
& \leq(n+1) v\left[T_{0}(r, W)+T_{0}(r, \widehat{W})\right]+O(1) .
\end{aligned}
$$

By the condition of Theorem 4.2, we know that the set of zeros of $W(z)-a_{j}$ and $\widehat{W}(z)-a_{j}$ in which each point counts only once, at the same time we get $\bar{N}_{12}\left(r, a_{j}\right)=0$.

Therefore

$$
\sum_{j=1}^{k-1} \bar{N}_{0}\left(r, \frac{1}{W^{(n)}-a_{j}}\right) \leq \sum_{j=1}^{k-1} \bar{N}_{0}\left(r, \frac{1}{W^{(n)}-\widehat{W}^{(n)}}\right) \leq(n+1) v\left[T_{0}(r, W)+T_{0}(r, \widehat{W})\right]+O(1) .
$$

From (4.15), (4.16) and (4.20), we have

$$
\sum_{j=1}^{k-1} \bar{N}_{0}\left(r, \frac{1}{W^{(n)}-a_{j}}\right) \leq\left(\frac{n+1}{k-(2 v+1)}+O(1)\right)\left[\sum_{j=1}^{k-1} \bar{N}_{0}\left(r, \frac{1}{W^{(n)}-a_{j}}\right)+\sum_{j=1}^{k-1} \bar{N}_{0}\left(r, \frac{1}{\widehat{W}^{(n)}-a_{j}}\right)\right]
$$

for $r \notin E$, which implies

$$
\left(\frac{k-(n+4 v)-\epsilon}{(k-2 v+1)-\epsilon}+O(1)\right) \sum_{j=1}^{k-1} \bar{N}_{0}\left(r, \frac{1}{W^{(n)}-a_{j}}\right) \leq\left(\frac{n+1}{k-(2 v+1)-\epsilon}+O(1)\right) \sum_{j=1}^{k-1} \bar{N}_{0}\left(r, \frac{1}{\widehat{W}^{(n)}-a_{j}}\right)
$$

for $r \notin E$.

Therefore, we obtain

$$
\varliminf_{r \rightarrow \infty} \frac{\sum_{j=1}^{k-1} \bar{N}_{0}\left(r, \frac{1}{W-a_{j}}\right)}{\sum_{j=1}^{k-1} \bar{N}_{0}\left(r, \frac{1}{\bar{W}-a_{j}}\right)} \leq \frac{n+1}{k-(n+4 v)-\epsilon}
$$

which is true for all $\epsilon>0$ and replace $k-1$ by $k$. Hence

$$
\varliminf_{r \rightarrow \infty} \frac{\sum_{j=1}^{k-1} \bar{N}_{0}\left(r, \frac{1}{\sum^{(n)}-a_{j}}\right)}{\sum_{j=1}^{k-1} \bar{N}_{0}\left(r, \frac{1}{\widehat{W}^{(n)}-a_{j}}\right)} \leq \frac{n+1}{k-(n+4 v)} .
$$

Where $a_{k}$ is finite (since all $a_{j}(1 \leq j \leq k)$ are finite). 
From (4.21) contradicts to (4.10) and hence $W^{(n)}(z) \equiv \widehat{W}^{(n)}(z)$. Now assume that one of the $a_{j}(1 \leq j \leq k)$ in (4.8) is infinity say $a_{k}=\infty$. Taking any finite value $a$ such that $a \neq a_{j}(1 \leq$ $j \leq k-1)$. Set

$$
F^{(n)}(z)=\frac{1}{W^{(n)}-a}, G^{(n)}(z)=\frac{1}{\widehat{W}^{(n)}-a} .
$$

Put $b_{j}=\frac{1}{a_{j}-a}(1 \leq j \leq k-1)$ and $b_{k}=0$.

Since $F^{(n)}(z)$ and $G^{(n)}(z)$ partially share finite values $b_{j}(1 \leq j \leq k-1)$ IM. Thus by the above case $F^{(n)}(z) \equiv G^{(n)}(z)$. Which completes the proof of theorem.

If $n=0$ in Theorem 4.2, then the conditions $E(0, W) \subseteq E\left(0, W^{(n)}\right)$ and $E(0, \widehat{W}) \subseteq E\left(0, \widehat{W}^{(n)}\right)$ are obvious and hence in this case, Theorem 4.2 reduces as follows

Theorem 4.3. Let $W(z)$ and $\widehat{W}(z)$ be two $v$-valued and $\mu$-valued algebroid functions on the annulus $\mathbb{A}\left(\frac{1}{R_{0}}, R_{0}\right)\left(1<R_{0} \leq+\infty\right)$, respectively and $\mu \leq v$, let $a_{j}(j=1,2, \ldots, k)$ be $k$ distinct small functions, where $k \geq 4 v+1$. If $\bar{E}\left(a_{j}, W\right) \subseteq \bar{E}\left(a_{j}, \widehat{W}\right)$ for all $1 \leq j \leq k$. If

$$
\frac{\varliminf_{r \rightarrow \infty} \sum_{j=1}^{k} N_{0}\left(r, \frac{1}{W-a_{j}}\right)}{\varliminf_{r \rightarrow \infty} \sum_{j=1}^{k} N_{0}\left(r, \frac{1}{\widehat{W}-a_{j}}\right)}>\frac{1}{k-(2 v+1)},
$$

then $W(z) \equiv \widehat{W}(z)$.

If $n=0$ and $k=4 v+1$ in Theorem 4.2, then Theorem 4.2 reduces as follows

Corollary 4.1. Let $W(z)$ and $\widehat{W}(z)$ be two $v$-valued and $\mu$-valued algebroid functions on the annulus $\mathbb{A}\left(\frac{1}{R_{0}}, R_{0}\right)\left(1<R_{0} \leq+\infty\right)$, respectively and $\mu \leq v$, let $a_{j}(j=1,2, \ldots, 4 v+1)$ be $4 v+1$ distinct small functions. If $\bar{E}\left(a_{j}, W\right) \subseteq \bar{E}\left(a_{j}, \widehat{W}\right)$ for all $1 \leq j \leq 4 v+1$, and

$$
\varliminf_{r \rightarrow \infty} \frac{\sum_{j=1}^{v+1} \bar{N}_{0}\left(r, \frac{1}{W-a_{j}}\right)}{\sum_{j=1}^{v+1} \bar{N}_{0}\left(r, \frac{1}{W-a_{j}}\right)}>\frac{1}{2 v},
$$

then $W(z) \equiv \widehat{W}(z)$.

Definition 4.2. Let $W(z)$ be an algebroid function on the annulus $\mathbb{A}\left(\frac{1}{R_{0}}, R_{0}\right)\left(1<R_{0} \leq+\infty\right)$ and $a(z)$ be a values of $W(z)$. We define

$$
\bar{W}(a, W)=\{z \mid W(z)-a(z)=0\}
$$

in which each zero is counted only once. 
We consider, two algebroid functions partially share five or more values on the annulus $\mathbb{A}\left(\frac{1}{R_{0}}, R_{0}\right)\left(1<R_{0} \leq+\infty\right)$. Precisely speaking, if two algebroid functions $W(z)$ and $\widehat{W}(z)$ on the annulus $\mathbb{A}\left(\frac{1}{R_{0}}, R_{0}\right)\left(1<R_{0} \leq+\infty\right)$ and $k$ be distinct values $a_{1}, a_{2}, \ldots, a_{k}, k \geq 4 v+1$ such that $\bar{E}\left(a_{j}, W\right) \subseteq \bar{E}\left(a_{j}, \widehat{W}\right)$, for all $1 \leq j \leq k$.

Now we can state and prove our theorem as follows

Theorem 4.4. Let $W(z)$ and $\widehat{W}(z)$ be two $v$-valued and $\mu$-valued algebroid functions on the annulus $\mathbb{A}\left(\frac{1}{R_{0}}, R_{0}\right)\left(1<R_{0} \leq+\infty\right)$, respectively and $\mu \leq v$, let $a_{j}(j=1,2, \ldots, k)$ be $k$ distinct values, where $k \geq 4 v+1$ and for a non negative integers $n$, if

$$
\begin{gathered}
E\left(a_{j}, W^{(n)}\right) \subseteq E\left(a_{j}, \widehat{W}^{(n)}\right), \quad \text { forall } 1 \leq j \leq k, \\
E\left(0, W_{1}\right) \subseteq E\left(0, W^{(n)}\right) \text { and } E(0, \widehat{W}) \subseteq E\left(0, \widehat{W}^{(n)}\right),
\end{gathered}
$$

and

$$
\frac{\varliminf_{r \rightarrow \infty} \sum_{j=1}^{k} N_{0}\left(r, \frac{1}{W^{(n)}-a_{j}}\right)}{\varliminf_{r \rightarrow \infty} \sum_{j=1}^{k} N_{0}\left(r, \frac{1}{\widehat{W}^{(n)}-a_{j}}\right)}>\frac{n+1}{k-(n+2 v+1)},
$$

then $W^{(n)}(z) \equiv \widehat{W}^{(n)}(z)$.

Proof. Using a similar argument as Theorem 4.2, we can prove it.

If $n=0$ in Theorem 4.4, then the conditions $E(0, W) \subseteq E\left(0, W^{(n)}\right)$ and $E(0, \widehat{W}) \subseteq E\left(0, \widehat{W}^{(n)}\right)$ are obvious and hence in this case, Theorem 4.4 reduces as follows

Theorem 4.5. Let $W(z)$ and $\widehat{W}(z)$ be two $v$-valued and $\mu$-valued algebroid functions on the annulus $\mathbb{A}\left(\frac{1}{R_{0}}, R_{0}\right)\left(1<R_{0} \leq+\infty\right)$, respectively and $\mu \leq v$, let $a_{j}(j=1,2, \ldots, k)$ be $k$ distinct values, where $k \geq 4 v+1$. If $\bar{E}\left(a_{j}, W\right) \subseteq \bar{E}\left(a_{j}, \widehat{W}\right)$ for all $1 \leq j \leq k$. If

$$
\frac{\varliminf_{r \rightarrow \infty} \sum_{j=1}^{k} N_{0}\left(r, \frac{1}{W-a_{j}}\right)}{\varliminf_{r \rightarrow \infty} \sum_{j=1}^{k} N_{0}\left(r, \frac{1}{\widehat{W}-a_{j}}\right)}>\frac{1}{k-(2 v+1)},
$$

then $W(z) \equiv \widehat{W}(z)$.

If $n=0$ and $k=4 v+1$ in Theorem 4.4, then Theorem 4.4 reduces as follows 
Corollary 4.2. Let $W(z)$ and $\widehat{W}(z)$ be two $v$-valued and $\mu$-valued algebroid functions on the annulus $\mathbb{A}\left(\frac{1}{R_{0}}, R_{0}\right)\left(1<R_{0} \leq+\infty\right)$, respectively and $\mu \leq v$, let $a_{j}(j=1,2, \ldots, 4 v+1)$ be $4 v+1$ distinct values. If $\bar{E}\left(a_{j}, W\right) \subseteq \bar{E}\left(a_{j}, \widehat{W}\right)$ for all $1 \leq j \leq 4 v+1$, and

$$
\varliminf_{r \rightarrow \infty} \frac{\sum_{j=1}^{v+1} \bar{N}_{0}\left(r, \frac{1}{W-a_{j}}\right)}{\sum_{j=1}^{v+1} \bar{N}_{0}\left(r, \frac{1}{\widehat{W}-a_{j}}\right)}>\frac{1}{2 v},
$$

then $W(z) \equiv \widehat{W}(z)$.

\section{Acknowledgement}

The author was supported by the UGC- Rajiv Gandhi National Fellowship (no. F1-17.1/201314-SC-KAR-40380) of India.

\section{References}

[1] E. Ullarich, Uber den Einfluess der verzweigtheit einer algebloide auf ihre wertvertellung, J Reine Angew Math, 169(1931), 198-220.

[2] G. Valiron, Sur quelques proprietes des fonctions algebroldes, Comptes Rendus Mathematique. Academie des Sciences. Paris, 189(1929), 824-826.

[3] N. Baganas, Sur les valeurs algebriques dune fonctions algebroldes et les integrales psendo-abelinnes, Annales Ecole Norm. Sup., 66(1949), 161-208.

[4] H. S. Gopalkrishna and S. S. Bhooanurmath, Uniqueness theorems for meromorphic functions, Math. Scand, 39(1976), 125-130.

[5] Yu-Zan He and Ye-Zhou Li., Some results on algebroid functions, Complex variables, 43 (2001), 299-313.

[6] S. Daochun and G. Zongsheng, On the operation of algebroid functions, Acta Mathematica Scientia, (2010), 247-256.

[7] S. Daochun and G. Zongsheng, Value Disribution Theory of Algebroid Functions, Beijing: Science Press, 2014.

[8] Yu-Zan He and Xiu-Zhi Xiao., Algebroid functions and Ordinarry Difference Equations, Science Press, Beijing, 1988.

[9] S. Daochun and G. Zongsheng, Theorems for algebroid functions, Acta Math. Sinica, 49 (2006), 1-6.

[10] Y. Hongxun, On the multiple values and uniqueness of algebroid functions , Eng.Math., 8 (1991), 1-8.

[11] W. K. Hayman, Meromorphic Functions, Oxford University Press, Oxford, 1964.

[12] F. Minglang, Unicity theorem for algebroid functions, Acta. Math. Sinica., 36 (1993), 217-222.

[13] Pingyuan Zhang and Peichu Hu, On uniqueness for algebroid functions of finite order, Acta. Math. Sinica., 35B (2015), 630-638.

[14] G. S. Prokoporich, Fix-points of meromorphic or entire functions, Ukrainian Math. J., 25 (1937), 248-260.

[15] Z. Qingcai, Uniqueness of algebroid functions, Math. Pract. Theory., 43 (2003), 183-187.

[16] Tingbin Cao and Hongxun Yi, On the uniqueness theory of algebroid functions, Southest Asian Bull. Math., 33(2009), 25-39.

[17] C. C. Yang and H. X. Yi, Uniqueness Theory of Meromorphic Functions, Science Press, 1995; Kluwer, 2003.

[18] H.X. Yi, The multiple values of meromorphic functions and uniqueness, Chinese Ann. Math. Ser. A, 10 (1989), 421-427. 
[19] Ashok Rathod, The multiple values of algebroid functions and uniqueness, Asian Journal of Mathematics and Computer Research, 14 (2016), 150-157.

[20] Ashok Rathod, Several uniqueness theorems for algebroid functions, J. Anal., DOI 10.1007/s41478-0041-x.

Department of Mathematics, Karnatak University, Dharwad - 580 003, India.

E-mail: ashokmrmaths@gmail.com 